\title{
On uniformly continuous Nemytskij operators generated by set-valued functions
}

\author{
EWELINA MAINKA
}

\begin{abstract}
Let $I=[0,1]$, let $Y$ be a real normed linear space, $C$ a convex cone in $Y$ and $Z$ a real Banach space. Denote by $c l b(Z)$ the set of all nonempty, convex, closed and bounded subsets of $Z$. If a superposition operator $N$ generated by a set-valued function $F: I \times C \rightarrow \operatorname{clb}(Z)$ maps the set $H_{\alpha}(I, C)$ of all Hölder functions $\varphi: I \rightarrow C$ into the set $H_{\beta}(I, \operatorname{clb}(Z))$ of all Hölder set-valued functions $\phi: I \rightarrow \operatorname{clb}(Z)$ and is uniformly continuous, then

$$
F(x, y)=A(x, y) \stackrel{*}{+} B(x), \quad x \in I, y \in C
$$

for some set-valued functions $A, B$ such that $A(\cdot, y), B \in H_{\beta}(I, \operatorname{clb}(Z)), y \in C$ and $A(x, \cdot)$, $x \in I$ are ${ }^{*}$ additive and continuous on $C$ into $\operatorname{clb}(Z)$. A converse result is also investigated.
\end{abstract}

Mathematics Subject Classification (2000). 47H04, 47H30, 54C60, 26E25.

Keywords. Jensen equation, set-valued functions, Nemytskij operator, uniformly continuous operators.

\section{Introduction}

Let $I, J \subset \mathbb{R}$ be intervals, $\alpha \in(0,1], x_{0} \in I$ and let $\operatorname{Lip}^{\alpha}(I, J)$ denote the set of all functions $\varphi: I \rightarrow J$ for which the set

$$
\left\{\frac{|\varphi(x)-\varphi(y)|}{|x-y|^{\alpha}}: x, y \in I, x \neq y\right\}
$$

is bounded with the functional

$$
\|\varphi\|_{L_{i p^{\alpha}}}:=\left|\varphi\left(x_{0}\right)\right|+\sup _{x, y \in I, x \neq y} \frac{|\varphi(x)-\varphi(y)|}{|x-y|^{\alpha}} .
$$

Each function $f: I \times J \rightarrow \mathbb{R}$ generates an operator of substitution (or Nemytskij operator) $N$ defined on $\operatorname{Lip}^{\alpha}(I, J)$ by the formula

$$
(N \varphi)(x)=f(x, \varphi(x)), \quad \varphi \in \operatorname{Lip}(I, J), x \in I .
$$


Recently Matkowski has shown (cf. [6]) that if a superposition operator $N$ of a generator $f$ maps the set $\operatorname{Lip}^{\alpha}(I, J)$ into the Banach space $\operatorname{Lip}^{\alpha}(I, \mathbb{R})$ and is uniformly continuous with respect to the norm (1), then

$$
f(x, y)=a(x) y+b(x), \quad x \in I, y \in J
$$

for some $a, b \in \operatorname{Lip}^{\alpha}(I, \mathbb{R})$. In this paper we are going to prove a counterpart of Matkowski's result for Nemytskij operators generated by set-valued functions with values in a set $\operatorname{clb}(Z)$ of all nonempty, bounded, closed, convex subsets of a normed linear space $Z$.

The problem of characterizing Lipschitzian operators of a substitution generated by set-valued functions with nonempty, compact, convex values, mapping the set of functions satisfying the Hölder condition into the set of such set-valued functions was investigated by Ludew (cf. [4]). Lipschitzian Nemytskij operators generated by set-valued functions with values in $c l b(Z)$ acting in the space of functions satisfying the Lipschitz condition were studied in [9].

Let $(Z,\|\cdot\|)$ be a real, normed linear space. For a bounded $A \subset Z$ one can define a number $\|A\|$ as follows $\|A\|:=\sup \{\|z\|: z \in A\}$.

We introduce a binary operation $\stackrel{*}{+}$ in $\operatorname{clb}(Z)$ by the formula

$$
A \stackrel{*}{+} B=\operatorname{cl}(A+B),
$$

where $A+B$ is an algebraic sum of $A$ and $B$ and $\operatorname{cl} A$ is the closure of $A$. Observe, that for arbitrary $A, B \in \operatorname{clb}(Z)$ the set $A+B$ does not have to be closed. A corresponding example can be found e.g. in $[10]$. The pair $(\operatorname{clb}(Z), \stackrel{*}{+})$ is an Abelian semigroup with the set $\{0\}$ as the zero element. We can multiply elements of $\operatorname{clb}(Z)$ by nonnegative numbers and the conditions

$$
1 \cdot A=A, \lambda(\mu A)=(\lambda \mu) A, \lambda(A \stackrel{*}{+} B)=\lambda A \stackrel{*}{+} \lambda B,(\lambda+\mu) A=\lambda A \stackrel{*}{+} \mu A
$$

hold for all $A, B \in \operatorname{clb}(Z)$ and $\lambda, \mu \geq 0$. This means that the set $\operatorname{clb}(Z)$ with operations $\stackrel{*}{+}$ and $\cdot$ is an abstract convex cone. The cancellation law, i.e.

$$
A \stackrel{*}{+} B=C \stackrel{*}{+} B \Longrightarrow A=C
$$

in $\operatorname{clb}(Z)$ follows e.g. from Theorem II-17 in [2, p. 48].

For two nonempty subsets $A, B$ of $Z$ and $z \in Z$ define

$$
\rho(z, B)=\inf \{\|z-b\|: b \in B\}, \quad e(A, B)=\sup \{\rho(a, B): a \in A\} .
$$

The Hausdorff distance $d(A, B)$ between $A$ and $B$ is defined by

$$
d(A, B)=\max \{e(A, B), e(B, A)\} .
$$

If $S$ denotes the closed unit ball in $Z$, then

$$
d(A, B)=\inf \{t>0: A \subset B+t S, B \subset A+t S\}
$$

for all nonempty and bounded sets $A$ and $B$. It is easily seen that $(\operatorname{clb}(Z), d)$ is a metric space. It is complete, provided $Z$ is a Banach space (cf. e.g. [2, p. 40]). 
It is easy to check that the Hausdorff distance is invariant with respect to translation, i.e.,

$$
d(A \stackrel{*}{+} B, C \stackrel{*}{+} B)=d(A+B, C+B)=d(A, C)
$$

(cf. e.g. [3]) and

$$
d(\lambda A, \lambda B)=|\lambda| d(A, B)
$$

for all $\lambda \geq 0$ and $A, B, C \in \operatorname{clb}(Z)$.

A subset $C$ of a real linear space $Y$ is said to be a convex cone if $\lambda C \subset C$ for all $\lambda \geq 0$ and $C+C \subset C$.

A set-valued function $F: C \rightarrow \operatorname{clb}(Z)$ defined on a convex cone $C$ is ${ }^{*} a d d i$ tive $\left({ }^{*}\right.$ Jensen $)$ if

$$
F(x+y)=F(x) \stackrel{*}{+} F(y) \quad\left(F\left(\frac{x+y}{2}\right)=\frac{1}{2}(F(x) \stackrel{*}{+} F(y))\right)
$$

for all $x, y \in C$. A function $F$ is $\mathbb{Q}_{+}$-homogeneous if $F(\lambda y)=\lambda F(y)$ for all $\lambda \in \mathbb{Q} \cap[0, \infty)$ and $y \in C$.

The following lemmas will be needed in the paper.

Lemma 1. (Corollary 4 in [9]) Let $C$ be a convex cone in a real linear space $Y$ and let $Z$ be a Banach space. A set-valued function $F: C \rightarrow \operatorname{clb}(Z)$ is ${ }^{*}$ Jensen if and only if there exist an ${ }^{*}$ additive set-valued function $A: C \rightarrow \operatorname{clb}(Z)$ and a set $B \in \operatorname{clb}(Z)$ such that

$$
F(x)=A(x) \stackrel{*}{+} B
$$

for $x \in C$.

Lemma 2. (Lemma 2 in [7]) Let $Y, Z$ be two real, normed linear spaces and let $C$ be a convex cone in $Y$. Suppose $F$ is a $\mathbb{Q}_{+}$-homogeneous set-valued function defined on $C$ with nonempty values in $Z$. The equality

$$
\lim _{y \rightarrow 0, y \in C}\|F(y)\|=0
$$

holds if and only if there exists a positive constant $M$ such that

$$
\|F(y)\| \leq M\|y\| \quad \text { for } y \in C .
$$

In the set of all $\mathbb{Q}_{+}$-homogeneous set-valued functions in $C$ with nonempty values in $Z$, satisfying (2) we can introduce the functional

$$
\|F\|=\sup _{x \in C, x \neq 0} \frac{\|F(x)\|}{\|x\|} .
$$

By Lemma 2, $\|F\|$ is finite. This functional will be called a norm. 
Lemma 3. (Lemma 4 and Remark 2 in [7]) Let $Y$ be a Banach space, $Z$ a real, normed linear space and let $C$ be a convex cone in $Y$. Suppose that $\left(F_{j}: j \in J\right)$ is a family of *additive, continuous set-valued functions $F_{j}: C \rightarrow$ clb $(Z)$. If int $C \neq \emptyset$ and for each $y \in C$ the set $\bigcup_{j \in J} F_{j}(y)$ is bounded in $Z$, then there exists a constant $M \in(0,+\infty)$ such that

$$
\sup _{j \in J}\left\|F_{j}\right\| \leq M \text {. }
$$

A function $\alpha:[0, \infty) \rightarrow[0, \infty)$ is said to be an $\alpha$-function, if $\alpha(t)>0$ for $t \in(0, \infty), \alpha(0)=0=\lim _{t \rightarrow 0} \alpha(t), \alpha(1)=1$ and both $\alpha$ and $\alpha^{*}$, where

$$
\alpha^{*}(t)= \begin{cases}\frac{t}{\alpha(t)} & \text { for } t \in(0, \infty) \\ 0 & \text { for } t=0\end{cases}
$$

are increasing (cf. [1, p. 182]).

Note that the function $\alpha(t)=t^{p}$, where $p \in(0,1]$ is an $\alpha$-function.

For two $\alpha$-functions $\alpha$ and $\beta$ we write

$$
\alpha \prec \beta \Longleftrightarrow \alpha(t)=O(\beta(t)) \quad \text { as } \quad t \rightarrow 0 .
$$

Let $\alpha$ be an $\alpha$-function, $I=[0,1]$ and let $C$ be a convex cone in a real, normed linear space Y. By definition, the set $H_{\alpha}(I, C)$ consists of all functions $\varphi: I \rightarrow C$ such that

$$
h_{\alpha}(\varphi):=\sup _{s \in(0,1]} \frac{\omega(\varphi, s)}{\alpha(s)}<+\infty
$$

where

$$
\omega(\varphi, s):=\sup \left\{|| \varphi\left(x_{1}\right)-\varphi\left(x_{2}\right) \|: x_{1}, x_{2} \in I,\left|x_{1}-x_{2}\right| \leq s\right\}
$$

(cf. [5]). By $H_{\alpha}(I, \operatorname{clb}(Z))$ we denote the set of all set-valued functions $\phi: I \rightarrow$ $\operatorname{clb}(Z)$ such that $h_{\alpha}(\phi)<+\infty$, where

$$
\omega(\phi, s):=\sup \left\{d\left(\phi\left(x_{1}\right), \phi\left(x_{2}\right)\right): x_{1}, x_{2} \in I,\left|x_{1}-x_{2}\right| \leq s\right\} .
$$

Observe that all functions from $H_{\alpha}(I, C)$ and from $H_{\alpha}(I, \operatorname{clb}(Z))$ are continuous. In fact, let us fix $x_{1}, x_{2} \in I$ and let $\varphi \in H_{\alpha}(I, C)$. We have

$$
\left\|\varphi\left(x_{1}\right)-\varphi\left(x_{2}\right)\right\| \leq \omega\left(\varphi,\left|x_{1}-x_{2}\right|\right) \leq h_{\alpha}(\varphi) \alpha\left(\left|x_{1}-x_{2}\right|\right) .
$$

Since $\alpha$ is continuous at $0,(5)$ shows that $\varphi$ is uniformly continuous, by (4). The same reasoning applies to $\phi \in H_{\alpha}(I, \operatorname{clb}(Z))$.

We introduce a metric $\rho_{\alpha}$ in the set $H_{\alpha}(I, C)$ putting $\rho_{\alpha}(\varphi, \bar{\varphi})=\|\varphi-\bar{\varphi}\|_{\alpha}$, where

$$
\|\varphi\|_{\alpha}:=\|\varphi(0)\|+h_{\alpha}(\varphi) .
$$

In the set $H_{\alpha}(I, \operatorname{clb}(Z))$ one can define a metric setting

$$
d_{\alpha}(\phi, \bar{\phi}):=d(\phi(0), \bar{\phi}(0))+\sup _{s \in(0,1]} \frac{\omega(\phi, \bar{\phi}, s)}{\alpha(s)}, \quad \phi, \bar{\phi} \in H_{\alpha}(I, \operatorname{clb}(Z)),
$$


where

$\omega(\phi, \bar{\phi}, s):=\sup \left\{d\left(\phi\left(x_{1}\right)+\bar{\phi}\left(x_{2}\right), \phi\left(x_{2}\right)+\bar{\phi}\left(x_{1}\right)\right): x_{1}, x_{2} \in I,\left|x_{1}-x_{2}\right| \leq s\right\}$

(cf. [8]). It may be proved that $d_{\alpha}(\phi, \bar{\phi})<\infty$ and that $d_{\alpha}$ is a metric in $H_{\alpha}(I, \operatorname{clb}(Z))$ (cf. [4]).

Consider the set

$$
\mathcal{L}(C, \operatorname{clb}(Z)):=\{A: C \rightarrow \operatorname{clb}(Z): A \text { is } * \text { additive and continuous }\} .
$$

Since every *additive set-valued function $A: C \rightarrow \operatorname{clb}(Z)$ is $\mathbb{Q}_{+}$-homogeneous, for each $A \in \mathcal{L}(C, \operatorname{clb}(Z))$ we have

$$
\|A(y)\| \leq\|A\|\|y\|, \quad y \in C
$$

where $\|A\|$ is defined by (3). Thus, for $A, B \in \mathcal{L}(C, \operatorname{clb}(Z))$ we have $d(A(y)$, $B(y)) \leq\|A(y)\|+\|B(y)\| \leq(\|A\|+\|B\|)\|y\|$ and

$$
d_{\mathcal{L}}(A, B):=\sup _{y \in C, y \neq 0} \frac{d(A(y), B(y))}{\|y\|}
$$

is finite. It is easily seen, that $d_{\mathcal{L}}$ yields a metric in $\mathcal{L}(C, \operatorname{clb}(Z))$.

\section{Main results}

Let $\alpha, \beta$ be $\alpha$-functions. We shall prove (in Theorem 2) that a uniformly continuous operator of a substitution $N$ mapping $H_{\alpha}(I, C)$ into $H_{\beta}(I, \operatorname{clb}(Z))$ has to be generated by a function $F: I \times C \rightarrow c l b(Z)$ of the form

$$
F(x, y)=A(x, y) \stackrel{*}{+} B(x)
$$

where $A(x, \cdot)$ is a *additive continuous set-valued function and $A(\cdot, y), B(\cdot)$ belong to $H_{\beta}(I, \operatorname{clb}(Z))$.

Theorem 1. Let $I=[0,1]$ and $Y$ be a real normed linear space, $Z$ a Banach space and let $C$ be a convex cone in $Y$. Assume that $\gamma:[0, \infty) \rightarrow[0, \infty)$ is continuous at $0, \gamma(0)=0$, and the superposition operator $N$ is generated by a set-valued function $F: I \times C \rightarrow \operatorname{clb}(Z)$.

(a) Suppose that $N$ maps $H_{\alpha}(I, C)$ into $H_{\beta}(I, \operatorname{clb}(Z))$ and

$$
d_{\beta}(N \varphi, N \bar{\varphi}) \leq \gamma\left(\|\varphi-\bar{\varphi}\|_{\alpha}\right), \quad \varphi, \bar{\varphi} \in H_{\alpha}(I, C) .
$$

Then there exist functions $A: I \times C \rightarrow \operatorname{clb}(Z)$ and $B: I \rightarrow \operatorname{clb}(Z)$ such that $A(\cdot, y), B \in H_{\beta}(I, \operatorname{clb}(Z))$ for every $y \in C, A(x, \cdot) \in \mathcal{L}(C, \operatorname{clb}(Z))$ for every $x \in I$ and

$$
F(x, y)=A(x, y) \stackrel{*}{+} B(x), \quad x \in I, y \in C .
$$


Moreover, the inequality

$d\left(A\left(x, y_{1}\right)+A\left(\bar{x}, y_{2}\right), A\left(\bar{x}, y_{1}\right)+A\left(x, y_{2}\right)\right) \leq \gamma\left(|| y_{1}-y_{2}||\right) \beta(|\bar{x}-x|)$

holds for all $x, \bar{x} \in I$ and $y_{1}, y_{2} \in C$.

(b) Assume that $\gamma$ is increasing and the condition $\frac{1}{\beta} \prec \gamma\left(\frac{1}{\alpha}\right)$ does not hold. Then the operator $N$ maps $H_{\alpha}(I, C)$ into $H_{\beta}(I, c l b(Z))$ and satisfies inequality (6) if and only if the function $F$ is of the form

$$
F(x, y)=B(x), \quad x \in I, y \in C,
$$

where $B \in H_{\beta}(I, \operatorname{clb}(Z))$. In this case $N$ is a constant operator.

Proof. (a) Note, that for a given $y \in C$ a constant function $\varphi(t)=y, t \in I$, belongs to the space $H_{\alpha}(I, C)$. Since $N$ maps $H_{\alpha}(I, C)$ into $H_{\beta}(I, c l b(Z))$, we have $N \varphi=F(\cdot, y) \in H_{\beta}(I, \operatorname{clb}(Z))$. Consequently, $F(\cdot, y)$ is continuous.

For arbitrarily fixed $y, \bar{y} \in C$, take $\varphi, \bar{\varphi}: I \rightarrow C$ defined by

$$
\varphi(t)=y, \quad \bar{\varphi}(t)=\bar{y}, \quad t \in I .
$$

Then $\varphi, \bar{\varphi} \in H_{\alpha}(I, C)$ and, by the assumption, functions $N \varphi=F(\cdot, y), N \bar{\varphi}=$ $F(\cdot, \bar{y})$ belong to $H_{\beta}(I, c l b(Z))$ and

$$
\|\varphi-\bar{\varphi}\|_{\alpha}=\|y-\bar{y}\| \text {. }
$$

From the definition of the metric $d_{\beta}$ we get

$$
d(N \varphi(0), N \bar{\varphi}(0))+\frac{\omega(N \varphi, N \bar{\varphi}, 1)}{\beta(1)} \leq d_{\beta}(N \varphi, N \bar{\varphi}) .
$$

Hence, by (6), for all $x \in I$

$$
d(F(0, y), F(0, \bar{y}))+d(F(x, y)+F(0, \bar{y}), F(x, \bar{y})+F(0, y)) \leq \gamma(\|y-\bar{y}\|) .
$$

Since

$$
\begin{aligned}
d(F(x, y), F(x, \bar{y}))= & d(F(x, y)+F(0, \bar{y}), F(x, \bar{y})+F(0, \bar{y})) \\
\leq & d(F(x, y)+F(0, \bar{y}), F(x, \bar{y})+F(0, y)) \\
& +d(F(x, \bar{y})+F(0, y), F(x, \bar{y})+F(0, \bar{y})) \\
= & d(F(0, y), F(0, \bar{y}))+d(F(x, y)+F(0, \bar{y}), F(x, \bar{y})+F(0, y)),
\end{aligned}
$$

(8) implies that

$$
d(F(x, y), F(x, \bar{y})) \leq \gamma(\|y-\bar{y}\|) \quad \text { for } \quad x \in I .
$$

This inequality, the continuity of $\gamma$ at 0 and the equality $\gamma(0)=0$ show that $F$ is continuous with respect to the second variable. 
Let us fix $x, \bar{x} \in I, x<\bar{x}, y_{1}, y_{2}, \bar{y}_{1}, \bar{y}_{2} \in C$ and define functions

$$
\varphi_{i}(t):= \begin{cases}y_{i} & \text { for } 0 \leq t \leq x, \\ \frac{\bar{y}_{i}-y_{i}}{\bar{x}-x}(t-x)+y_{i} & \text { for } x<t<\bar{x} \\ \bar{y}_{i} & \text { for } \bar{x} \leq t \leq 1\end{cases}
$$

for $i=1,2$. Obviously, $\varphi_{i}(I) \subseteq C$. We shall prove that $\varphi_{i} \in H_{\alpha}(I, C)$. It is easy to see that

$$
\begin{array}{r}
\omega\left(\varphi_{i}, s\right)=\left\|\bar{y}_{i}-y_{i}\right\| \text { for } \bar{x}-x \leq s \leq 1, \\
\omega\left(\varphi_{i}, s\right)=\frac{s}{\bar{x}-x}\left\|\bar{y}_{i}-y_{i}\right\| \text { for } 0 \leq s \leq \bar{x}-x .
\end{array}
$$

Since the function $t \longmapsto \frac{t}{\alpha(t)}$ is increasing

$$
\sup _{s \in(0,1]} \frac{\omega\left(\varphi_{i}, s\right)}{\alpha(s)}=\frac{\left\|\bar{y}_{i}-y_{i}\right\|}{\alpha(\bar{x}-x)} .
$$

Therefore, $\varphi_{i} \in H_{\alpha}(I, C)$ and $\left\|\varphi_{i}\right\|_{\alpha}=\left\|y_{i}\right\|+\frac{\left\|\bar{y}_{i}-y_{i}\right\|}{\alpha(\bar{x}-x)}$. In particular

$$
\left\|\varphi_{1}-\varphi_{2}\right\|_{\alpha}=\left\|y_{1}-y_{2}\right\|+\frac{\left\|\bar{y}_{1}-\bar{y}_{2}-y_{1}+y_{2}\right\|}{\alpha(\bar{x}-x)} .
$$

From (6) and the definition of $d_{\beta}$

$$
\frac{\omega\left(N \varphi_{1}, N \varphi_{2}, \bar{x}-x\right)}{\beta(\bar{x}-x)} \leq d_{\beta}\left(N \varphi_{1}, N \varphi_{2}\right) \leq \gamma\left(\left\|\varphi_{1}-\varphi_{2}\right\|_{\alpha}\right)
$$

and since $\varphi_{i}(x)=y_{i}$ and $\varphi_{i}(\bar{x})=\bar{y}_{i}$,

$$
d\left(F\left(x, y_{1}\right)+F\left(\bar{x}, \bar{y}_{2}\right), F\left(\bar{x}, \bar{y}_{1}\right)+F\left(x, y_{2}\right)\right) \leq \gamma\left(\left\|\varphi_{1}-\varphi_{2}\right\|_{\alpha}\right) \beta(\bar{x}-x) .
$$

Taking arbitrary $u, v \in C$ and setting $y_{1}=\bar{y}_{2}=\frac{u+v}{2}, \bar{y}_{1}=u, y_{2}=v$ we obtain

$$
\left\|\varphi_{1}-\varphi_{2}\right\|_{\alpha}=\frac{\|u-v\|}{2}
$$

and

$d\left(F\left(x, \frac{u+v}{2}\right)+F\left(\bar{x}, \frac{u+v}{2}\right), F(\bar{x}, u)+F(x, v)\right) \leq \gamma\left(\frac{\|u-v\|}{2}\right) \beta(\bar{x}-x)$.

Letting $\bar{x}$ tend to $x$, since $\lim _{t \rightarrow 0} \beta(t)=0$, from the continuity of $F$ with respect to the first variable we get

$$
d\left(2 F\left(x, \frac{u+v}{2}\right), F(x, u)+F(x, v)\right)=0
$$

i.e.

$$
F\left(x, \frac{u+v}{2}\right)=\frac{1}{2}[F(x, u) \stackrel{*}{+} F(x, v)]
$$


for all $x \in I$. This shows, that $F(x, \cdot)$ is *Jensen, which implies that there exist functions $A: I \times C \rightarrow \operatorname{clb}(Z)$ and $B: I \rightarrow \operatorname{clb}(Z)$ such that $A(x, \cdot)$ is *additive for $x \in I$ and

$$
F(x, y)=A(x, y) \stackrel{*}{+} B(x), \quad x \in I, y \in C
$$

(cf. Lemma 1).

To prove that $A(x, \cdot)(x \in I)$ is continuous let us fix $y, \bar{y} \in C$. We have

$$
\begin{aligned}
d(A(x, y), A(x, \bar{y})) & =d(A(x, y) \stackrel{*}{+} B(x), A(x, \bar{y}) \stackrel{*}{+} B(x)) \\
& =d(F(x, y), F(x, \bar{y})),
\end{aligned}
$$

therefore, the continuity of $F(x, \cdot)$ implies the continuity of $A(x, \cdot)$.

From the *additivity of $A(x, \cdot)$ we have $A(x, 0)=\{0\}$, whence

$$
F(x, 0)=A(x, 0) \stackrel{*}{+} B(x)=B(x) .
$$

Since $F(\cdot, y) \in H_{\beta}(I, \operatorname{clb}(Z))$ for all $y \in C,(12)$ shows that $B \in H_{\beta}(I, \operatorname{clb}(Z))$.

Now we shall prove that $A(\cdot, y) \in H_{\beta}(I, c l b(Z))$ for every $y \in C$. Let us fix $s \in(0,1], x, \bar{x} \in I$ such that $|x-\bar{x}| \leq s$ and $y \in C$. Of course

$$
\begin{aligned}
d(A(x, y), A(\bar{x}, y))= & d(A(x, y)+B(x), A(\bar{x}, y)+B(x)) \\
\leq & d(A(x, y)+B(x), A(\bar{x}, y)+B(\bar{x})) \\
& +d(A(\bar{x}, y)+B(\bar{x}), A(\bar{x}, y)+B(x)) \\
= & d(F(x, y), F(\bar{x}, y))+d(B(x), B(\bar{x})),
\end{aligned}
$$

whence

$$
d(A(x, y), A(\bar{x}, y)) \leq \omega(F(\cdot, y), s)+\omega(B, s)
$$

and

$$
\frac{\omega(A(\cdot, y), s)}{\beta(s)} \leq h_{\beta}(F(\cdot, y))+h_{\beta}(B) .
$$

The fact that $A(\cdot, y) \in H_{\beta}(I, \operatorname{clb}(Z))$ for every $y \in C$ follows now from the inequality above.

Finally, to show (7) take $x, \bar{x} \in I$ such that $x<\bar{x}$ and $y_{1}, y_{2} \in C$. Putting $\bar{y}_{1}=y_{1}, \bar{y}_{2}=y_{2}$ in (9) and (10) we obtain

$$
d\left(F\left(x, y_{1}\right)+F\left(\bar{x}, y_{2}\right), F\left(\bar{x}, y_{1}\right)+F\left(x, y_{2}\right)\right) \leq \gamma\left(\left\|y_{1}-y_{2}\right\|\right) \beta(\bar{x}-x) .
$$

Therefore

$$
\begin{gathered}
d\left(A\left(x, y_{1}\right)+B(x)+A\left(\bar{x}, y_{2}\right)+B(\bar{x}), A\left(\bar{x}, y_{1}\right)+B(\bar{x})+A\left(x, y_{2}\right)+B(x)\right. \\
\quad=d\left(A\left(x, y_{1}\right)+A\left(\bar{x}, y_{2}\right), A\left(\bar{x}, y_{1}\right)+A\left(x, y_{2}\right)\right) \leq \gamma\left(\left\|y_{1}-y_{2}\right\|\right) \beta(\bar{x}-x)
\end{gathered}
$$

The obtained inequality

$$
d\left(A\left(x, y_{1}\right)+A\left(\bar{x}, y_{2}\right), A\left(\bar{x}, y_{1}\right)+A\left(x, y_{2}\right)\right) \leq \gamma\left(|| y_{1}-y_{2} \|\right) \beta(|\bar{x}-x|)
$$

for all $y_{1}, y_{2} \in C$ and $x, \bar{x} \in I, x<\bar{x}$ is also true in the case of $x \geq \bar{x}$, which completes the proof of part (a). 
(b) It suffices to show necessity. Setting $y_{1}=y_{2}$ in (9) and (10) we obtain

$$
d\left(F\left(\bar{x}, \bar{y}_{1}\right), F\left(\bar{x}, \bar{y}_{2}\right)\right) \leq \gamma\left(\frac{\left\|\bar{y}_{1}-\bar{y}_{2}\right\|}{\alpha(\bar{x}-x)}\right) \beta(\bar{x}-x)
$$

for all $x, \bar{x} \in I$ such that $x<\bar{x}$ and for all $\bar{y}_{1}, \bar{y}_{2} \in C$. In the case $\left\|\bar{y}_{1}-\bar{y}_{2}\right\| \leq 1$ by the monotonicity of $\gamma$ we have

$$
d\left(F\left(\bar{x}, \bar{y}_{1}\right), F\left(\bar{x}, \bar{y}_{2}\right)\right) \leq \gamma\left(\frac{1}{\alpha(\bar{x}-x)}\right) \beta(\bar{x}-x) .
$$

Since the condition $\frac{1}{\gamma\left(\frac{1}{\alpha}\right)} \prec \beta$ does not hold, we can find a sequence $\left(t_{n}\right), t_{n} \in$ $(0,1], t_{n} \rightarrow 0$, such that

$$
\beta\left(t_{n}\right) \gamma\left(\frac{1}{\alpha\left(t_{n}\right)}\right) \rightarrow 0 \quad \text { as } n \rightarrow \infty .
$$

Take $x \in[0,1)$ and $\bar{x}_{n}:=x+t_{n}$. Then $\bar{x}_{n} \in[0,1]$ for a large enough $n$ and $\bar{x}_{n} \rightarrow x$. Since $F(\cdot, y), y \in C$ is continuous, from (13) and (14) we deduce that $F\left(x, \bar{y}_{1}\right)=F\left(x, \bar{y}_{2}\right), x \in[0,1]$ and $\bar{y}_{1}, \bar{y}_{2} \in C$.

In the case $\left\|\bar{y}_{1}-\bar{y}_{2}\right\|>1$, fix $n$ large enough to have $\frac{1}{n}\left\|\bar{y}_{1}-\bar{y}_{2}\right\| \leq 1$. Setting $y^{i}=\bar{y}_{1}+\frac{i}{n}\left(\bar{y}_{2}-\bar{y}_{1}\right), i=0,1, \ldots, n-1$, we obtain $\left\|y^{i+1}-y^{i}\right\| \leq 1$. By the above, we get $F\left(x, y^{i}\right)=F\left(x, y^{i+1}\right)$ for all $x \in I$ and $i=0,1, \ldots, n-1$, whence $F\left(x, \bar{y}_{1}\right)=F\left(x, \bar{y}_{2}\right)$ for all $x \in I$ and $\bar{y}_{1}, \bar{y}_{2} \in C$. Consequently, $F(x, y)=$ $F(x, 0)=: B(x)$ for $x \in I$ and $y \in C$, which completes the proof.

Remark 1. Denote by $\mathcal{A}$ the set of all functions $\varphi \in H_{\alpha}(I, C)$ of the form

$$
\varphi(t):= \begin{cases}y & \text { for } 0 \leq t \leq x, \\ \frac{\bar{y}-y}{\bar{x}-x}(t-x)+y & \text { for } x<t<\bar{x} \\ \bar{y} & \text { for } \bar{x} \leq t \leq 1\end{cases}
$$

for some $x, \bar{x} \in I, x<\bar{x}, y, \bar{y} \in C$. From the argument used in the proof follows that Theorem 1 remains valid if inequality (6) is postulated only for all $\varphi, \bar{\varphi} \in \mathcal{A}$.

Remark 2. Assuming, that $\gamma$ in Theorem 1 is increasing does not cause any loss of generality.

Indeed, for a given $\gamma:[0, \infty) \rightarrow[0, \infty)$ we can take $\gamma^{*}:[0, \infty) \rightarrow[0, \infty)$ defined by $\gamma^{*}(t)=\sup _{s \in[0, t]} \gamma(s)$.

Condition (6) in Theorem 1 can be replaced by the uniform continuity of $N$.

Theorem 2. Let $Y$ be a real normed linear space, $Z$ a Banach space and $C$ a convex cone in $Y$. Suppose that the superposition operator $N$ of the generator $F: I \times C \rightarrow \operatorname{clb}(Z)$ maps $H_{\alpha}(I, C)$ into $H_{\beta}(I, c l b(Z))$ and that $N$ is uniformly continuous. Then there exist functions $A: I \times C \rightarrow \operatorname{clb}(Z)$ and $B: I \rightarrow \operatorname{clb}(Z)$ 
such that $A(\cdot, y), B \in H_{\beta}(I, \operatorname{clb}(Z))$ for every $y \in C, A(x, \cdot) \in \mathcal{L}(C, \operatorname{clb}(Z))$ for every $x \in I$ and

$$
F(x, y)=A(x, y) \stackrel{*}{+} B(x), \quad x \in I, \quad y \in C .
$$

Proof. Suppose that $N$ is uniformly continuous. Then for every $\varepsilon>0$ there is $\delta>0$ such that for all $\varphi, \bar{\varphi} \in H_{\alpha}(I, C)$

$$
\|\varphi-\bar{\varphi}\|_{\alpha} \leq \delta \Longrightarrow d_{\beta}(N \varphi, N \bar{\varphi}) \leq \varepsilon .
$$

Let $\gamma:[0, \infty) \rightarrow[0, \infty)$ be defined by the formula

$$
\gamma(t):=\sup \left\{d_{\beta}(N \varphi, N \bar{\varphi}):\|\varphi-\bar{\varphi}\|_{\alpha} \leq t\right\}, \quad t \geq 0 .
$$

The function $\gamma$ is well defined. Indeed, fix $\delta>0$ such that for all $\varphi, \bar{\varphi} \in$ $H_{\alpha}(I, C)$

$$
\|\varphi-\bar{\varphi}\|_{\alpha} \leq \delta \Longrightarrow d_{\beta}(N \varphi, N \bar{\varphi}) \leq 1 .
$$

Hence, we have $\gamma(t) \leq 1$ for all $t \in[0, \delta]$. Take $t \geq 0, s \geq 0, t+s>0$ and $\varphi, \bar{\varphi} \in H_{\alpha}(I, C)$ such that $\|\varphi-\bar{\varphi}\|_{\alpha} \leq t+s$. The function $\psi=\frac{t}{t+s} \varphi+\frac{s}{t+s} \bar{\varphi}$ also belongs to $H_{\alpha}(I, C)$ and

$$
\|\varphi-\psi\|_{\alpha}=\frac{s}{t+s}\|\varphi-\bar{\varphi}\|_{\alpha} \leq s, \quad\|\psi-\bar{\varphi}\|_{\alpha}=\frac{t}{t+s}\|\varphi-\bar{\varphi}\|_{\alpha} \leq t .
$$

Thus, by the definition of $\gamma$ it follows that

$$
d_{\beta}(N \varphi, N \bar{\varphi}) \leq d_{\beta}(N \varphi, N \psi)+d_{\beta}(N \psi, N \bar{\varphi}) \leq \gamma(s)+\gamma(t)
$$

and consequently

$$
\gamma(s+t) \leq \gamma(s)+\gamma(t) .
$$

In particular, $\gamma(2 t) \leq 2 \gamma(t)$, whence by induction we obtain

$$
\gamma(n t) \leq n \gamma(t)
$$

for all $n \in \mathbb{N}$ and $t \geq 0$. For a given $t \geq 0$ there exists a positive integer $n$ such that $\frac{t}{n}<\delta$. From (15) and (16) it follows that

$$
\gamma(t)=\gamma\left(n \frac{t}{n}\right) \leq n \gamma\left(\frac{t}{n}\right) \leq n<\infty .
$$

Since $N$ is uniformly continuous, $\gamma$ is continuous at $0, \gamma(0)=0$ and obviously

$$
d_{\beta}(N \varphi, N \bar{\varphi}) \leq \gamma\left(\|\varphi-\bar{\varphi}\|_{\alpha}\right), \quad \varphi, \bar{\varphi} \in H_{\alpha}(I, C),
$$

thus the result is a consequence of Theorem 1 .

Remark 3. If in Theorem $1, \gamma(t)=L t$ (for some constant $L \geq 0$ ) and the function $F$ maps $I \times C$ into the space $c c(Z)$ of all nonempty, convex and compact subsets of $Z$, we can replace $\stackrel{*}{+}$ by the usual algebraic sum of two sets and we get the result obtained by Ludew in [4]. 


\section{A converse result}

The following result may be proved in the same way as Lemma 5 in [7].

Lemma 4. Let $Y$ and $Z$ be two real, normed linear spaces and $C$ a convex cone in $Y$ with nonempty interior. Then there exists a positive constant $M_{0}$ such that for every continuous, ${ }^{*}$ additive, set-valued function $F: C \rightarrow \operatorname{clb}(Z)$ the inequality

$$
d(F(x), F(y)) \leq M_{0}\|F\|\|x-y\|, \quad x, y \in C
$$

holds.

The following theorem is a converse of part (a) of Theorem 1.

Theorem 3. Let $Y$ be a Banach space, $Z$ a real normed linear space, $C$ a convex cone in $Y$ with nonempty interior and let $\alpha, \beta$ be two $\alpha$-functions such that $\alpha \prec \beta$. Assume that $A(\cdot, y), B \in H_{\beta}(I, \operatorname{clb}(Z))$ for $y \in C$ and $A(x, \cdot) \in$ $\mathcal{L}(C, \operatorname{clb}(Z))$ for $x \in I$. Moreover, assume that for some increasing, continuous at 0 function $\gamma:[0, \infty) \rightarrow[0, \infty)$, such that $\gamma(0)=0$, the inequality

$$
d\left(A\left(x, y_{1}\right)+A\left(\bar{x}, y_{2}\right), A\left(\bar{x}, y_{1}\right)+A\left(x, y_{2}\right)\right) \leq \gamma\left(|| y_{1}-y_{2}||\right) \beta(|\bar{x}-x|)
$$

holds for all $x, \bar{x} \in I$ and $y_{1}, y_{2} \in C$. If a set-valued function $F: I \times C \rightarrow \operatorname{clb}(Z)$ is of the form

$$
F(x, y)=A(x, y) \stackrel{*}{+} B(x), \quad x \in I, \quad y \in C,
$$

then the operator of the substitution $N$ generated by $F$ maps the set $H_{\alpha}(I, C)$ into $H_{\beta}(I, c l b(Z))$ and satisfies inequality (6) with a function $\gamma_{1}$, where $\gamma_{1}(t)=$ $c(t+\gamma(t)), t \in[0, \infty)$ and $c$ is a constant.

Proof. First, we shall prove that for an arbitrary $y \in C$ the set $\bigcup_{x \in I} A(x, y)$ is bounded. Let $x \in I, y \in C$. We have

$$
\begin{aligned}
\|A(x, y)\| & =d(A(x, y),\{0\}) \leq d(A(x, y), A(0, y))+d(A(0, y),\{0\}) \\
& =d(A(x, y), A(0, y))+\|A(0, y)\| .
\end{aligned}
$$

Moreover, since $A(\cdot, y) \in H_{\beta}(I, \operatorname{clb}(Z))$,

$$
d(A(x, y), A(0, y)) \leq \omega(A(\cdot, y), 1)=\frac{\omega(A(\cdot, y), 1)}{\beta(1)} \leq h_{\beta}(A(\cdot, y))<\infty .
$$

Therefore

$$
\|A(x, y)\| \leq h_{\beta}(A(\cdot, y))+\|A(0, y)\|, \quad x \in I .
$$

Since $\{A(x, \cdot)\}_{x \in I}$ is a family of *additive and continuous functions, by Lemma 3 there exists a constant $M>0$ such that

$$
\sup _{x \in I}\|A(x, y)\| \leq M\|y\|, \quad y \in C .
$$


Hence, and by Lemma 4, we deduce that

$$
d(A(x, y), A(x, \bar{y})) \leq M_{0} M\|y-\bar{y}\|
$$

for all $x \in I$ and $y, \bar{y} \in C$.

We shall prove now that $N$ maps $H_{\alpha}(I, C)$ into $H_{\beta}(I, \operatorname{clb}(Z))$. Let $\varphi \in$ $H_{\alpha}(I, C)$ and $x, \bar{x} \in I$. The inequality

$$
d(A(x, y), A(\bar{x}, y)) \leq \gamma(|| y||) \beta(|\bar{x}-x|)
$$

is a consequence of (17). From (18) and (19) we get

$$
\begin{aligned}
d(N \varphi(x), N \varphi(\bar{x}))= & d(A(x, \varphi(x))+B(x), A(\bar{x}, \varphi(\bar{x}))+B(\bar{x})) \\
\leq & d(A(x, \varphi(x)), A(\bar{x}, \varphi(\bar{x})))+d(B(x), B(\bar{x})) \\
\leq & d(A(x, \varphi(x)), A(\bar{x}, \varphi(x))) \\
& +d(A(\bar{x}, \varphi(x)), A(\bar{x}, \varphi(\bar{x})))+d(B(x), B(\bar{x})) \\
\leq & \gamma(\|\varphi(x)\|) \beta(|\bar{x}-x|)+M_{0} M\|\varphi(x)-\varphi(\bar{x})\|+d(B(x), B(\bar{x}))
\end{aligned}
$$

for all $x, \bar{x} \in I$. Since

$$
\|\varphi(x)\| \leq\|\varphi(0)\|+\frac{\|\varphi(x)-\varphi(0)\|}{\alpha(x-0)} \alpha(x-0), \quad x \in(0,1]
$$

we have $\|\varphi(x)\| \leq\|\varphi\|_{\alpha}$ for every $x \in I$. Now let us take $s \in(0,1]$ and $x, \bar{x} \in I$ such that $|x-\bar{x}| \leq s$. Due to the monotonicity of $\gamma$ and $\beta$

$$
d(N \varphi(x), N \varphi(\bar{x})) \leq \gamma\left(\|\varphi\|_{\alpha}\right) \beta(s)+M_{0} M \omega(\varphi, s)+\omega(B, s) .
$$

Thus, for every $s \in(0,1]$ we obtain

$$
\begin{aligned}
\frac{\omega(N \varphi, s)}{\beta(s)} & \leq \gamma\left(\|\varphi\|_{\alpha}\right)+M_{0} M \frac{\omega(\varphi, s)}{\alpha(s)} \frac{\alpha(s)}{\beta(s)}+\frac{\omega(B, s)}{\beta(s)} \\
& \leq \gamma\left(\|\varphi\|_{\alpha}\right)+L M_{0} M h_{\alpha}(\varphi)+h_{\beta}(B),
\end{aligned}
$$

where $L>1$ is some constant such that $\frac{\alpha(s)}{\beta(s)} \leq L, s \in(0,1]$ (by the assumption $\alpha \prec \beta)$. Therefore, $h_{\beta}(N \varphi)<\infty$ and $N \varphi \in H_{\beta}(I, c l b(Z))$.

It remains to show that $N$ satisfies $(6)$. Let $\varphi, \bar{\varphi} \in H_{\alpha}(I, C), s \in(0,1]$ and take $x, \bar{x} \in I$ such that $|\bar{x}-x| \leq s$. Inequalities (18) and (17) imply that

$$
\begin{aligned}
d( & N \varphi(x)+N \bar{\varphi}(\bar{x}), N \varphi(\bar{x})+N \bar{\varphi}(x)) \\
= & d(A(x, \varphi(x))+A(\bar{x}, \bar{\varphi}(\bar{x})), A(\bar{x}, \varphi(\bar{x}))+A(x, \bar{\varphi}(x))) \\
= & d(A(x, \varphi(x)+\bar{\varphi}(\bar{x}))+A(\bar{x}, \bar{\varphi}(\bar{x}))+A(x, \varphi(\bar{x})) \\
A(x, \varphi(\bar{x})+\bar{\varphi}(x))+A(x, \bar{\varphi}(\bar{x}))+A(\bar{x}, \varphi(\bar{x}))) & \quad d(A(x, \varphi(x)+\bar{\varphi}(\bar{x}), A(x, \varphi(\bar{x})+\bar{\varphi}(x))) \\
\quad \leq & +d(A(\bar{x}, \bar{\varphi}(\bar{x}))+A(x, \varphi(\bar{x})), A(x, \bar{\varphi}(\bar{x}))+A(\bar{x}, \varphi(\bar{x}))) \\
& \leq M_{0} M\|(\varphi-\bar{\varphi})(x)-(\varphi-\bar{\varphi})(\bar{x})\|+\gamma(\|(\varphi-\bar{\varphi})(\bar{x})\|) \beta(|\bar{x}-x|) \\
\leq & M_{0} M \omega(\varphi-\bar{\varphi}, s)+\gamma\left(\|\varphi-\bar{\varphi}\|_{\alpha}\right) \beta(s) .
\end{aligned}
$$


Hence,

$$
\frac{\omega(N \varphi, N \bar{\varphi}, s)}{\beta(s)} \leq M_{0} M \frac{\omega(\varphi-\bar{\varphi}, s)}{\alpha(s)} \frac{\alpha(s)}{\beta(s)}+\gamma\left(\|\varphi-\bar{\varphi}\|_{\alpha}\right)
$$

and therefore,

$$
\sup _{s \in(0,1]} \frac{\omega(N \varphi, N \bar{\varphi}, s)}{\beta(s)} \leq M_{0} M L h_{\alpha}(\varphi-\bar{\varphi})+\gamma\left(\|\varphi-\bar{\varphi}\|_{\alpha}\right) .
$$

Consequently,

$$
\begin{aligned}
d_{\beta}(N \varphi, N \bar{\varphi}) & =d(N \varphi(0), N \bar{\varphi}(0))+\sup _{s \in(0,1]} \frac{\omega(N \varphi, N \bar{\varphi}, s)}{\beta(s)} \\
& \leq M_{0} M\|(\varphi-\bar{\varphi})(0)\|+M_{0} M L h_{\alpha}(\varphi-\bar{\varphi})+\gamma\left(\|\varphi-\bar{\varphi}\|_{\alpha}\right) \\
& \leq M_{0} M L\|\varphi-\bar{\varphi}\|_{\alpha}+\gamma\left(\|\varphi-\bar{\varphi}\|_{\alpha}\right) .
\end{aligned}
$$

Setting $c=\max \left\{1, M_{0} M L\right\}$ and $\gamma_{1}(t)=c(t+\gamma(t))$ we get

$$
d_{\beta}(N \varphi, N \bar{\varphi}) \leq \gamma_{1}\left(\|\varphi-\bar{\varphi}\|_{\alpha}\right) .
$$

This finishes the proof.

Open Access. This article is distributed under the terms of the Creative Commons Attribution Noncommercial License which permits any noncommercial use, distribution, and reproduction in any medium, provided the original author(s) and source are credited.

\section{References}

[1] Appel, J., Zabrejko, P.P.: Nonlinear Superposition Operators. Cambridge University Press, Cambridge (1990)

[2] Castaing, C., Valadier, M.: Convex analysis and measurable multifunctions. In: Lecture Notes in Mathematics, vol. 580 (1977)

[3] De Blasi, F.S.: On differentiability of multifunctions. Pac. J. Math. 66, 67-81 (1976)

[4] Ludew, J.J.: On Lipschitzian operators of substitution generated by set-valued functions. Opscula Math. 27(1), 13-24 (2007)

[5] Matkowski, J.: Lipschitzian composition operators in some function spaces. Nonlinear Anal. Theory Methods Appl. 30(2), 719-726 (1997)

[6] Matkowski, J.: Uniformly continuous superposition operators in the space of Hölder functions. J. Math. Anal. Appl. 359, 56-61 (2009)

[7] Smajdor, A.: On regular multivalued cosine families. Ann. Math. Sil. 13, 271-280 (1999)

[8] Smajdor, A., Smajdor, W.: Jensen equation and Nemytskii operator for set-valued functions. Rad. Math. 5, 311-320 (1989)

[9] Smajdor, W.: Note on Jensen and Pexider functional equations. Demonstratio Math. 32(2), 363-376 (1999)

[10] Smajdor, W.: Note on a Jensen type functional equation. Publ. Math. Debrecen 63(4), 703-714 (2003) 
Ewelina Mainka

Institute of Mathematics

Silesian University of Technology

Kaszubska 23

44-100 Gliwice

Poland

e-mail: Ewelina.Mainka@polsl.pl

Received: September 20, 2009

Revised: May 1, 2010 